\title{
Una mirada a las enfermedades parasitarias en el país
}

\author{
Claudia L. Sánchez C. MSc. \\ Instituto de Salud Publica, Universidad Nacional de Colombia. \\ Correpondencia: claudialsanchezc@yahoo.com
}

El concepto de salud ha evolucionado de manera significativa, pasando de una interpretación basada en lo biológico que ubica en un extremo la enfermedad y en el otro la salud como no enfermedad; a una donde se entiende como un fenómeno estrechamente ligado a las condiciones generales de vida de la población (1) donde intervienen diversos factores o determinantes relacionados con el bienestar: acceso a servicios, vivien$\mathrm{da}$, entre otros. En este orden de ideas teniendo en cuenta los factores que intervienen en el bienestar o salud, el desequilibrio puede contribuir a la aparición de algunas enfermedades parasitarias.

Las enfermedades parasitarias hacen parte de la historia de la humanidad es así como algunos autores señalan asociaciones parasitarias encontradas en restos fósiles de foraminíferos (protozoos con concha calcárea) y algas marinas con más de 530,000,000 años de antigüedad. Los parásitos se pueden encontrar en diferentes organismos ya sea animales o plantas. El momento exacto del hallazgo de los parásitos en el hombre tiene su origen en tiempos remotos y han sido descritos por diversas culturas tanto por los griegos, los egipcios y hasta los chinos (2)

Precisamente, fueron los chinos quienes describieron en detalle cuadros clínicos febriles representativos de la malaria, la presencia de gusanos como la Taenia $s p$. y sus respectivos tratamientos.

Es por ello que se puede afirmar que los parásitos han coexistido con la humanidad y por ello se puede hablar de su efecto e impacto a nivel salud y en el sector económico de una región.

Sí bien existen diferentes enfermedades parasitarias; algunas son un problema importante de salud publica por la morbi - mortalidad que causan y en algunos casos por su dificultad para controlarlas, a nivel mundial la malaria, esquistosomiasis,
Leishmaniasis, enfermedad de Chagas y las parasitosis intestinales son ejemplo de ellas. Nuestro país también se ve afectado por algunas de estas enfermedades, a continuación se describe brevemente la situación:

\section{Parasitismo intestinal:}

En Colombia según la Investigación Nacional de Morbilidad realizada entre 1965 y 1980, se reportó el comportamiento de los parásitos patógenos de la siguiente manera: Ascaris lumbricoides, en 1965 se encontraba en un 54\% y disminuyó en 1980 a un 34\%. Trichuris trichiura de un 50\% reportado en 1965, en 1980 disminuyó a un 37\%. El complejo Entamoeba histolytica/ dispar, en 1965 se reportó con una prevalencia de $24 \%$ y disminuyó al $12 \%$ en 1980 . Giardia lamblia, al contrario de los parásitos anteriormente nombrados, se comportó de manera ascendente en el tiempo, aumentando su prevalencia de 9,4\% reportada en 1965 a un $21,4 \%$ en 1980 (3).

\section{Malaria:}

La malaria representa un grave problema de salud en el $85 \%$ del territorio rural colombiano situado por debajo de los 1600 m.s.n.m con condiciones climáticas, 


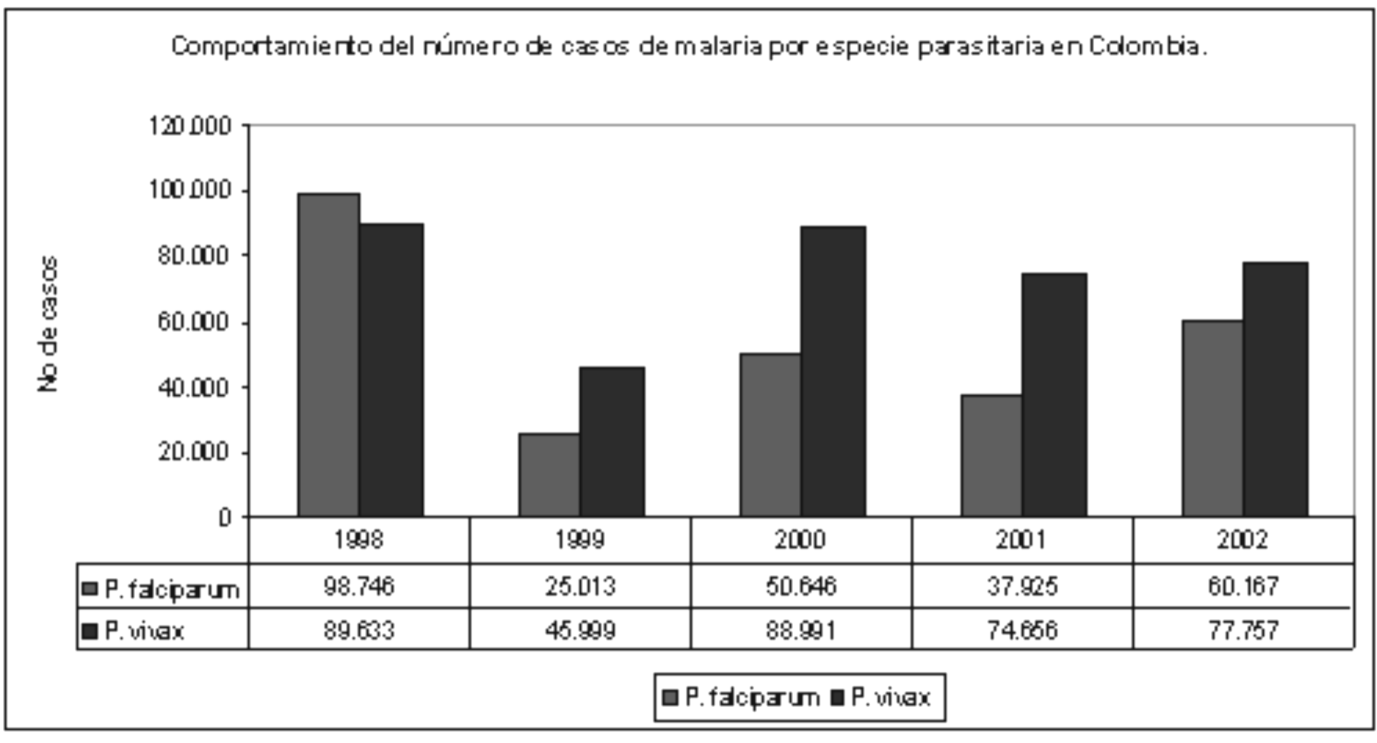

Figura 1. Total de casos de Malaria por P. Falciparum y P. Vivax. Colombia 1998 a 2002

geográficas y epidemiológicas aptas para la transmisión de la enfermedad, estimándose que entre 18 a 24 millones de personas se encuentran en riesgo de enfermar o morir por esta causa (4)

Especial relevancia ha adquirido la infección causada por P. falciparum, la cual ha aumentando desde 1996 alcanzando en 1998, la mayor tasa de incidencia de las últimas dos décadas. El alto número de casos debidos a $P$. falciparum, podría explicar el incremento de la incidencia de la malaria, toda vez que simultáneamente se ha registrado aumento de la resistencia de esta especie a los medicamentos antimaláricos de primera línea usados en el país, Figura 1. En 1997 se registraron en el país 180.910 casos de malaria, $36,5 \%$ de ellos causados por P. falciparum y $63 \%$ por P. vivax, lo que equivale a una incidencia general de 7.1 casos por cada 1.000 habitantes de zonas de riesgo. En 1998, se notificaron 194.178 casos de malaria, para una tasa de 7.5 por 1000 habitantes de zonas de riesgo. La distribución por especie fue de 50.7\% por P. falciparum, $47.8 \%$ por P. vivax y $1.5 \%$ por malaria asociada. Es de resaltar que el $45 \%$ de los casos notificados corresponden al departamento del Chocó (5).

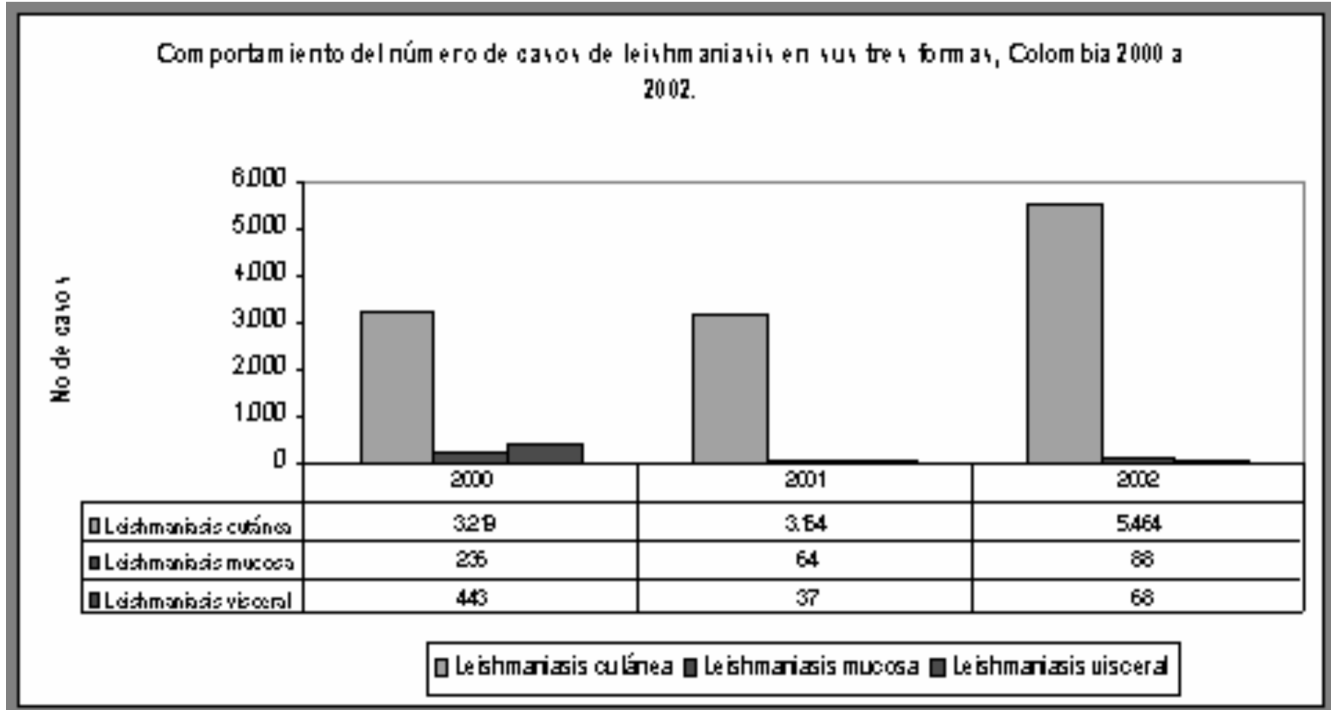

Figura 2. Comportamiento del número de casos de leishmaniasis en sus tres formas, Colombia 2000 a 2002. 


\section{Leishmaniasis:}

El total de casos reportados al SIVIGILA en el año 2000 , fue de 3.898 casos de leishmaniasis en todas sus formas, en 2001 fueron informados 3.265 casos y en el 2002, 5.620 casos de los cuales el 97.2\% (5.464) corresponden a casos de leishmaniasis cutánea (6), Figura 2.

\section{Enfermedad de Chagas:}

Existen alrededor de 8.000 .000 personas, en Colombia que están expuestas a la transmisión vectorial de la Enfermedad de Chagas y se estima entre 700.000 y 1.200.000 las personas infectadas en el país (7). El estudio nacional de seroprevalencia y factores de riesgo de la enfermedad de Chagas, realizado en una muestra representativa de la población y las viviendas, encontró una prevalencia de infección chagásica de 35 por 1.000 niños menores de 15 años, principalmente en la región oriental del país (8). Esto nos indica que, en esta región, cerca de 37.500 niños pueden estar afectados por la enfermedad.

La transmisión vectorial representa un problema de salud pública en los departamentos de Arauca (6.1/1.000), Casanare (23.8/1.000), Norte de Santander (10.9/1.000), Santander (3.9/1.000) y Boyacá (7.2/1.000). El riesgo de transmisión vectorial está focalizado en 137 municipios distribuidos en los referidos departamentos.

Se han realizado estudios puntuales de morbilidad en población adulta con el objetivo de determinar la frecuencia y gravedad de la cardiopatía en poblaciones de individuos chagásicos no seleccionados en Boyacá, Santander y grupos indígenas de la Sierra nevada de Santa Marta encontrándose una seropositividad entre 19.4 a $47 \%$ y alteraciones electrocardiográficas entre 25 y $47.8 \%(8,9)$.

\section{Las parasitosis y su impacto}

Las enfermedades parasitarias constituyen un problema importante de salud pública en países en vía de desarrollo como el nuestro y es común a los diferentes grupos de edad, su población blanco es la niñez y evaluándose en términos de muerte prematura y en Años de Vida Potencial Perdidos (AVPP) constituye una importante pérdida para cualquier país.

El impacto de estas enfermedades en la carga de la enfermedad es variado, en el caso de las parasitosis intestinales se relaciona con efectos negativos en el crecimiento, el desarrollo motor y cognoscitivo y la aparición de anemia (10) .

En Colombia este tipo de enfermedades se ven favorecidas por las condiciones geográficas, climáticas y socio económicas manteniendo la prevalencia a lo largo del tiempo. No es novedoso afirmar que las parasitosis son enfermedades de los pobres y que la mayor parte de nuestra población sufre o sufrió de alguna de estas enfermedades.

La alta incidencia de los casos de estas enfermedades puede imponer una elevada carga económica a las familias y los países por la disminución de la productividad, la pérdida de posibilidades educativas y los altos costos. Es innegable también que mientras no haya cambios estas condiciones: mala o insuficiente alimentación, condiciones de vivienda precaria, falta de saneamiento o saneamiento inadecuado, carencia de agua potable o malas condiciones de aprovisionamiento de agua, falta de higiene y mala preparación de los alimentos no habrá disminución en la prevalencia de estas parasitosis.

Por ello es necesario distribuir los recursos con que se cuentan e invertir los medios existentes en campañas eficaces desde diferentes puntos de acción: saneamiento ambiental y en campañas de capacitación a la población (ya esta plenamente documentado que lavarse las manos antes de comer, así como lavar frutas y verduras disminuyen considerablemente las parasitosis intestinales) para informarlos y tratar de involucrar otros estamentos como el de la educación dado que su colaboración sería prioritaria para la disminución de dichas patologías.

www.unicolmayor.edu.co 


\section{Referencias}

1. Quevedo E. El proceso Salud - Enfermedad: Hacía una clínica y una epidemiología no positivistas. Zeus editores.1992. P. 38 .

2. Organización Mundial de la Salud. Situación de Salud en las Américas. 2000.

3. Corredor A, Arciniegas E, Hernández CA. Parasitismo Intestinal. Bogotá: Ministerio de Salud, Instituto Nacional de Salud; 2002. p.90.

4. Ministerio de la Protección Social. Instituto Nacional de Salud. Informe de Situación del Programa Nacional de Vigilancia y Control de la Malaria en Colombia durante el año 2001; Bogotá

5. Informe Quincenal Epidemiólogico. Ministerio de Protección social. Instituto Nacional de Salud. 2005;10 (3): 37-43

6. Informe Quincenal Epidemiólogico. Ministerio de Protección social. Instituto Nacional de Salud. 2004; 9 (14): 209-212
7. PAHO. Health Conditions in the Americas. Volume I. Washington DC, 1990.

8. Nodo Universidad de los Andes, CIMPAT. Informe final de la primera fase del proyecto Nacional de Prevención y Control de la Enfermedad de Chagas y la Cardiopatía Infantil. Presentado al Ministerio de Salud. Bogotá: Universidad de los Andes; 1999.p.340

9. Guhl, F, Restrepo, M , Angulo, VM, Antunes,CM, CampbellLendrum, D and Davies C. (2005). Lessons from a nacional survey of Chagas disease transmisión risk in Colombia.TRENDS in Parasitology 21, (6):259-262

10. Smith H, Kaminsky R, Niwas S, Soto R, Jolly P. Prevalence and intensity of infections of Ascaris lumbricoides and Trichuris trichiura and associated socio-demographic variables in four rural Honduran communities. Mem Inst Oswaldo Cruz 2001;96:303-14. 